\title{
Review of Management of Gastrointestinal Stromal Tumor in Low-Resource Centers
}

\author{
Seke Manase Ephraim Kazuma, Bright Chirengendure, Patrick Musonda, Joseph Musowoya, Royd \\ Ngoma, Boniface Kaela, Simunyama Luyando, and Kamwi Mundia
}

\section{ABSTRACT}

Gastrointestinal stromal tumors (GIST) account for $1 \%$ to $3 \%$ of gastrointestinal tract tumors and are the most common of the mesenchymal tumors. Carcinogenesis of GIST arises in the interstitial cells of Cajal (ICC) and in the myenteric plexus of the gastrointestinal tract due to a mutation of the kinase receptor (KIT, also known as CD117) and the platelet-derived growth factor A (PDGFA) gene leading to activation of the tyrosine kinase receptor. The exact incidence and prevalence of GIST is not known. Symptoms of GIST are non-specific; they present with GI bleeding due to ulceration $(50 \%)$, abdominal pain $(20 \%$ to $50 \%)$, dysphagia (esophageal GIST) and GI obstruction $(10 \%$ to $30 \%)(7,10)$. Signs include abdominal mass and fullness. A computerized tomographic (CT) scan is the preferred imaging to evaluate GIST. Diagnosis is confirmed by immunohistochemical (IHC) staining a of biopsy sample for medical treatment tyrosine kinase inhibitors (TKI). Surgical resection with negative microscopic margins is the gold standard treatment of GIST. TKI are required for tumor reduction to increase chances of respectability (neoadjuvant therapy) or to prevent recurrence and reduce the progression of advanced, resectable GIST.

Keywords: CD117, GIST, gastrointestinal stromal tumor, KIT, TKI.

Published Online: November 17, 2021

ISSN: $2736-5476$

DOI: 10.24018 /ejclinicmed.2021.2.6.140

S. M. E. Kazuma*

Department of Surgery, Ndola Teaching Hospital, Ndola, Zambia.

(e-mail: sekekazuma@gmail.com)

B. Chirengendure

Department of Surgery, Ndola Teaching

Hospital, Ndola, Zambia.

P. Musonda

Department of Surgery, Ndola Teaching

Hospital, Ndola, Zambia.

J. Musowoya

Department of Surgery, Ndola Teaching Hospital, Ndola, Zambia.

R. Ngoma

Department of Surgery, Ndola Teaching Hospital, Ndola, Zambia.

B. Kaela

Department of Surgery, Ndola Teaching Hospital, Ndola, Zambia.

S. Luyando

Department of Surgery, Ndola Teaching

Hospital, Ndola, Zambia.

K. Mundia

Department of Surgery, Ndola Teaching

Hospital, Ndola, Zambia.

*Corresponding Author

\section{INTRODUCTION}

Gastrointestinal stromal tumors (GIST) account for $1 \%$ to $3 \%$ of gastrointestinal tract tumors and $5 \%$ to $6 \%$ of sarcomas and are the most common subset of mesenchymal tumors [1][3]. GIST has an annual incidence of 10-20 million people per year worldwide [4], [5]. GIST arises in the submucosa of the gastrointestinal (GI) tract but can also arise outside the GI tract, from the mesentery and greater omentum (extravisceral) [6]-[8]. Carcinogenesis of GIST arises in the interstitial cells of Cajal (ICC) and in the myenteric plexus of the GI due to a mutation of the kinase receptor (KIT, also known as CD117) and the platelet-derived growth factor A (PDGFA) gene, leading to activation of the tyrosine kinase receptor [9], [10]. In histology, the 3 types of GIST include spindle cell type $(70 \%)$, epithelioid type $(20 \%)$ and mixed type $(10 \%)$ [10], [11].

GIST is usually asymptomatic and/or incidentally diagnosed during surgery or on imaging [12], [13]. Clinical presentation and management of GIST depend on size and location of the tumor and its clinical features [14]. Although GIST can arise anywhere in the GI, 70\% are gastric, $25 \%$ are small intestinal, $5 \%$ to $10 \%$ are in the colon and rectum, $5 \%$ are in the esophagus and $10 \%$ are extravisceral (8).

GIST can appear at any age; median age is 60 years [7], [13]. Age is an important risk factor for GIST [7]. The risk of GIST increases with an increase in mutations, which can be inherited and found in several members of one family [7]. Since $95 \%$ of GIST are positive for CD117, diagnosis is based on a positive stain of KIT (CD117) on immunohistochemical (IHC) staining [15], [16]. Surgical resection with clear margins for resectable GIST and for GIST with resectable oligometastasis can be liver or peritoneal metastasis [12], [17].

The aim of this paper is to discuss the management of GIST in a low-resource center. 


\section{History AND EVOLUTION OF GIST}

Between 1930 and 1950, stromal tumors were considered to arise from smooth muscle, based on light microscopic features, and were classified as leiomyoma, leiomyosarcoma or leiomyoblastoma [18]-[20]. In 1983, Muzur and Clark [21] introduced the term stromal tumor and were recognized for introducing the term with supporting evidence that these originated from the GI tract and were a distinct entity [22], [23]. In the mid-1990s, these tumors were discovered to be positive for CD34, a relatively specific marker for diagnosis [24]. In 1998, KIT gene mutation was discovered by [25]. PDGFA was discovered by [26]. The pro-growth signal that leads to development of GIST was identified by [27]. In 2004, DOG, a calcium-dependence chloride channel protein anoctamin found in GIST regardless of the presence of CD117 and PDGFA, was discovered [28]; 95\% of GIST stain positive for CD117 and DOG [28].

\section{EPIDEMIOLOGY OF GIST}

Though the exact incidence and prevalence of GIST is not known, it occurs at all ages, and 90\% occur in those older than 40 years of age [4], [29]. The incidence in males and females is the same; however, a slight male predominance has been observed [28]. Geographical location, race and occupation have no attributed risk to the development of GIST [4], [29]. Between $20 \%$ and $30 \%$ of GIST are malignant and can present with metastatic disease [4]. Most GIST is sporadic, with familial cases reported at $1.4 \%$ to $4 \%(4,29)$. Familial GIST with autosomal dominance inheritance has been described [29]. Familial GIST includes neurofibromatosis, Carney-Stratakis syndrome and Carney triad. Reference [30] describe the Carney triad as composed of gastric GIST, extraadrenal paraganglioma and pulmonary chondroma. However, a Carney triad needs to be distinguished from inherited GIST syndrome with paragangliomas, called Carney-Stratakis syndrome [29].

\section{ClinicAl Features}

Seventy percent of GIST are symptomatic, $20 \%$ asymptomatic and 10\% discovered at autopsy [7], [13]. Though symptoms of GIST are non-specific, they present with GI bleeding due to ulceration (50\%), abdominal pain (20\% to $50 \%$ ), dysphagia (esophageal GIST) and GI obstruction (10\% to $30 \%)$ [7], [10]. Signs include abdominal mass and fullness [10]. Some GIST present with paraneoplastic symptoms of hypoglycemia due to the effect of an insulin-like growth factor produced [10]. GIST frequently metastasis to the liver $(65 \%)$, the peritoneum through hematogenous metastasis $(25 \%)$ and less frequently to the omentum, but rarely through lymphatic metastasis to regional lymph nodes and non-GI organs [31]. Hematogenous metastasis to organs outside the abdomen occurs late in advanced disease [13]. GIST tends to grow bigger and displace other organs without invading neighboring organs [13]. Reference [32] reported GI bleeding and abdominal pain at $58 \%$ and $60 \%$, respectively.

Small GIST, up to $2 \mathrm{~cm}$, are usually asymptomatic, discovered incidentally during surgical interventions or surveillance for other conditions, until symptoms develop due to enlarging size [14]. Large GIST, of more than $5 \mathrm{~cm}$, present with obstruction or bleeding [2], [33]. Half of GIST are metastatic at presentation [7]. Life-threatening haemorrhagic peritonitis may result from a ruptured GIST [4], [7].

\section{PATHOPhysiology OF GIST}

GIST carcinogenesis arises from the muscularis propria smooth muscle pacemaker cells known as ICC. The role of ICC is to coordinate GI peristalsis due to mutation of the transmembrane protein, a tyrosine kinase receptor. This receptor's intracellular (juxtamembrane) domain stimulates cell proliferation and cell hyperfunction and inhibits apoptosis, a protooncogene called the KIT (CD117) gene (in $95 \%$ of patients) or the PDGFA (in $30 \%$ of patients lacking KIT gene mutation) [10], [28], [34], [35]. While DOG1 is present in $95 \%$ of GIST, even in the absence of KIT and PDGFA, its role in the diagnosis of GIST is still uncertain [28]. Mutation of the KIT gene leads to uncontrolled and enhanced cell proliferation [13], [28], [29]. Most mutations are deletion, duplication or point mutations that can be primary (original cell mutations) or secondary (because of mutations arising from treatment) [3], [13], [29]. The most common mutations are in exon 11 (the most frequent malignant outcome), while rare mutations are in exon 9 and exon 13 [13].

Histological subtypes of GIST include 4 composed of an epithelioid pattern and another 4 composed of spindle cells [3], [28], [33]. Histological subtypes include the following:

1. sclerosing epithelioid variant (polyhedral cells, collagenous stroma deposition, multinucleated and low mitotic rate),

2. dyscohesive epithelioid variant (polygonal cells, halo surrounded cells, mild nuclear pleomorphism),

3. hypercellular epithelioid variant (highly neoplastic with low mitotic rate),

4. sarcomatous epithelioid variant (high cellularity with high mitotic rate),

5. sclerosing spindle cell variant (hypocellularity, collagenous stroma and focal calcifications),

6. palisading vacuolated spindle variant (commonly gastric location, spindle- and schwannoma-like cells, prominent nuclear vacuolation with low mitotic rate),

7. hypercullular spindle variant (dense cell atypia with low mitotic rate), and

8. sarcomatous spindle variant (karyomegaly, nuclear hyperchromatism with high mitotic rate).

\section{IMPORTANCE OF IMAGING}

The imaging of choice for diagnosing and evaluating the response to treatment and follow-up of primary and metastatic GIST is contrast-enhanced computer tomography (CECT) [13], [28]. Magnetic resonance imaging has a comparable yield, adds the advantage of lack of radiation and is accurate for diagnosis [2], [10]. A fluorodeoxyglucose positron emission tomography (FDG-PET) scan is used to obtain an initial diagnosis and for evaluating the presence of metastatic disease and response to treatment but is not readily 
available in low-resource countries due to cost and nonavailability in health facilities [13], [33]. A lack of metabolic response on an FDG-PET scan indicates primary resistance to treatment by tyrosine kinase inhibitors (TKI), while reoccurrence of metabolic activity indicates development of secondary resistance [33].

\section{DIAGNOSIS OF GIST}

Initial evaluation should start with a thorough history and physical examination, followed by imaging to evaluate the primary tumor and presence of metastasis [10]. Preoperative tissue diagnosis is not recommended in resectable tumors strongly suspected to be GIST, but tissue diagnosis may be required in advanced and unresectable GIST, requiring neoadjuvant therapy, or in metastatic GIST, requiring palliative adjuvant therapy with TKI [7], [33]. Tissue for histopathology can be obtained by endoscopic ultrasound, where available, or image-guided percutaneous biopsy [4], [28], [33]. Pathological diagnosis is supported by tumor morphology and IHC staining of the tissue obtained either by en-bloc excision or fine-needle aspiration cytology (FNAC) $(2,10)$. FNAC can be obtained by image guidance (ultrasound or endoscopic) [4], [28], [33]. Diagnosis is confirmed by the presence of CD117 on IHC [7], [13], [28]. GIST should be a differential diagnosis for any non-epithelial abdominal tumor [28], [29].

\section{MANAGEMENT OF GIST}

The standard treatment of resectable GIST, which is considered curative, is wide surgical resection with microscopic negative surgical margins (R0) and is considered the gold standard treatment for $80 \%$ of GIST [4], [6], [7], [28]. Tumor adherence to neighboring organs or invasion of adjacent organs, mitotic index and tumor size are important factors that guide treatment choice [7], [28]. Unresectable GIST, defined as GIST that is locally advanced, metastatic 6 or too big to achieve a negative resection margin, is treated with neoadjuvant TKI or adjuvant TKI [7], [15], [28]. According to the National Comprehensive Cancer Network (NCCN) guidelines, GIST smaller than $2 \mathrm{~cm}$ should be managed conservatively, with follow-up by imaging and endoscopic ultrasound guided (EUS) FNAC to assess the risk of malignancy [28], [36]. Any size GIST with high risk of malignancy should be treated by complete surgical resection [7], [36]. The risk of GIST is assessed by mitotic index (with mitoses more than 5 mitoses/50 high power field [HPF]) and an increase in tumor size; these are associated with a worse outcome [15], [36].

High-risk GIST is defined by mitotic rate, tumor size, tumor perforation at surgery and primary organ of origin [6], [7], [29]. According to Miettinen's criteria [6], [7]:

1. GIST smaller than $5 \mathrm{~cm}$ and fewer than 5 mitoses $/ 50 \mathrm{HPF}$ are low-risk;

2. GIST larger than $5 \mathrm{~cm}$ and more than 5 mitoses $/ 50 \mathrm{HPF}$ are high-risk;

3. gastric GIST up to $10 \mathrm{~cm}$ and 5 mitoses $/ 50 \mathrm{HPF}$ are lowrisk, while larger than $10 \mathrm{~cm}$ and more than 5 mitoses $/ 50$ HPF are high-risk;
4. all intestinal GIST larger than $5 \mathrm{~cm}$ and more than 5 mitoses $/ 50 \mathrm{HPF}$ are high-risk, while smaller than $5 \mathrm{~cm}$ and fewer than 5 mitoses/50 HPF are low-risk.

\section{Medical Management}

Treatment with TKI is recommended in GIST that are resectable but with significant morbidity, borderline resectable (to shrink the tumor and increase negative resection margins), locally advanced (invasion of adjacent organs), metastatic or recurring [1], [6], [26]. TKI can be used in neoadjuvant and adjuvant therapy. Gene sequencing of a biopsy or FNAC sample is required before treatment with medical therapy (neoadjuvant or adjuvant therapy) to determine tumor response as mutation to PDGFA; $10 \%$ of other mutations show no response to TKI [7], [12]. However, there is no established criteria to measure tumor response [17].

The TKI available for medical treatment of GIST include imatinib for TKI mutation or avapritinib for PDGFA mutations (first line) and suratinab (second line, because it it inhibits many KIT, PDGFA and vascular endothelial growth factor alpha tyrosine kinases), with no other regimen if these two TKI fail [13], [28], [36]. Patients with undetectable KIT, PDGFA or known mutations resistant to TKI may not benefit from treatment with TKI [2].

\section{A. Neoadjuvant Therapy}

Neoadjuvant therapy is required to achieve negative resection margins and lowers surgical morbidity (by reducing extent of resection and lowering bleeding at surgery) [14], [28]. Neoadjuvant therapy is also indicated in organ-sparing or function -paring surgery such as esophagectomy for esophageal GIST [28]. NCCN guidelines recommend neoadjuvant therapy for those with resectable disease but with significant morbidity, borderline resectable disease, resectable recurrent or metastatic disease [36]. Neoadjuvant therapy is recommended for 4 to 6 months, but such treatment should be decided on individual basis [36]. Imaging is used to detect the response to neoadjuvant therapy [12], [13], [33]. Reference [4] reports that reduced tumor size and vascularization as a response to neoadjuvant therapy result in reduced chances of tumor rupture and peritoneal implantation. Some studies have reported unexpected, good responses in metastatic GIST to neoadjuvant therapy, with downsizing of GIST from unresectable to resectable [4], [12].

\section{B. Adjuvant Therapy}

TKI is recommended in those with GIST that are high-risk, resectable with tumor rupture, unresectable, recurrent, or metastatic [2], [29], [36], [37]. Imatinib has been recommended as adjuvant treatment after surgical resection because it has the benefit of reducing recurrence after surgery [36], [37]. Imatinib can also be used in palliative treatment of GIST [36], [37]. Resected GIST without adjuvant treatment with TKI have an overall survival (OS) of $35 \%$ at 5 years [29]. Adjuvant therapy is continued for 3 years to reduce tumor recurrence [29], [36]. Reference [29] reports a 5-year OS of $54 \%$ in those with advanced GIST, with 19 months median survival, and 12 months in those with recurrent disease. Reference [4] reports $50 \%$ response to imatinib in a 
prospective trial; $50 \%$ to $75 \%$ had stable disease, while 2 -year survival after imatinib was $70 \%$.

\section{Surgical Treatment}

Currently, surgical resection is the gold standard and aims at achieving a negative resection margin (en-bloc resection of local disease including involved adjacent organs) without tumor rupture [6], [7], [36]. Surgical resection of primary localized disease offers a 5 -year OS of $48 \%$ to $70 \%$ (7). Reference [29] reports that complete resection is associated with a 5 -year OS of $42 \%$, and $19 \%$ if resection is incomplete. Formal lymph node dissection is not advised by current guidelines [13], [36]. Lymph node metastasis is not common in GIST, and most studies have reported negative lymph nodes after dissection [14], [36]. However, [14] reports occurrence of a positive lymph node after total gastrectomy with distal pancreatectomy and splenectomy for a fundal GIST in a patient who received neoadjuvant therapy. This patient did not have recurrent disease after 12 months of adjuvant therapy and after 24 months follow-up [14]. Palliative surgery may be done for control of symptoms or complications (bleeding or compression of adjacent organs) [4], [36].

Surgical resection with microscopic negative margins can be achieved by open surgery (especially for large GIST) or minimally invasive surgery (laparoscopic or robotic) [7], [29]. Low-resource centers tend to perform open surgery due to the cost of laparoscopic or robotic surgery [7], [13], [29].

\section{Prognosis}

All GISTs are considered malignant until proven otherwise, even though tumor mitotic index and tumor size are the most important prognostic factors [4], [13]. Reference [38] investigated mortality in GIST smaller than $2 \mathrm{~cm}$ and found mortality rates of $34 \%$ in regionally advanced GIST, $34.4 \%$ in metastatic GIST and 5.5\% in localized GIST. GIST cannot be considered benign simply on account of small size [36], [38]. Reference [1] reports a 3-year OS after surgery for locally advanced at $87 \%$, while recurrent or metastatic disease had an OS of $80 \%$.

\section{Outcome}

Complete resection without tumor rupture avoids peritoneal seeding, intra-abdominal tumor recurrence and tumor recurrence in a surgical scar, while liver metastasis is hematogenously spread [7], [12], [13]. Complete resection with negative margins has a 5-years OS of $60 \%$ [12]. Complete resected GIST has a liver recurrence of $65 \%$, peritoneal recurrence of $50 \%$ and both liver and perineal recurrence of $20 \%$ [4]. Though microscopic negative resection margins have a 5 -year OS of $48 \%$ to $65 \%$, OS is determined by tumor size and mitotic index and not by negative resection margins [4]. High-risk GIST should receive neoadjuvant or adjuvant therapy with imatinib to improve outcome. Reference [33] reports that in a study with 170 patients who underwent complete resection with microscopic negative margins and adjuvant therapy with imatinib, median survival was 7.3 years to 8.7 years.

\section{Follow-UP}

The outcome of GIST is unpredictable, and therefore long follow-up is required [4], [36], [37]. Follow-up should be done with history and physical exam every 3 to 4 months for 2 years, then every 6 months for 2 years, and yearly afterwards [36], [37]. NCCN guidelines recommend followup with imaging (CT/CT-PET) every 3 to 6 months for 3 to 5 years, and then yearly thereafter [36]. Blood tests can be done yearly [36], [37].

\section{CONCLUSION}

GIST are rare, non-epithelial GIT tumors that arise from ICC with an unpredictable clinical course and high chances of malignancy. The preferred imaging for GIST is CECT scan. Preoperative diagnosis is not required for resectable GIST, except where neoadjuvant therapy for borderline resectable or resected high-risk GIST, or if the tumor is unresectable and requires adjuvant therapy. Tumor size and mitotic index are the most important prognostic factors in the management of GIST.

\section{ACKNOWLEDGMENT}

We thank the management of Ndola Teaching Hospital for the support.

\section{FUNDING}

None.

\section{CONFLICT OF INTEREST}

None.

\section{ETHICS APPROVAL}

Not applicable.

\section{REFERENCES}

[1] Bischof DA, Kim Y, Blazer DG, Behman R, Karanicolas PJ, Law CH, et al. Surgical management of advanced gastrointestinal stromal tumors: An international multi-institutional analysis of 158 patients. Journal of the American College Surgeons. 2014; 219: 439-449.

[2] Lamba G, Gupta R, Lee B, Ambrale S, Liu D. Current management and prognostic features for gastrointestinal stromal tumor (GIST). Experimental Hematology and Oncology. 2012; 1(1): 14.

[3] Boşoteanu M, Boşoteanu C, Deacu M, Aşchie M. Differential diagnosis of a gastric stromal tumor: case report and literature review. Romanian Journal of Morphology and Embryology. 2011; 52(4): 1361-1368.

[4] Michael S, Emmanouel D, Charikleia S, Panagiotis S, Electra P, Georgia L, et al. Gastrointestinal stromal tumor. World Journal of Surgical Oncology. 2009; 7: 61.

[5] Liu P, Tan F, Liu H, Ge J, Liu S, Lei T, et al. Skin metastasis of gastrointestinal stromal tumors: a case series and literature review. Cancer Management and Research. 2020; 12: 7681.

[6] Yasemin A, Mehmet B. Gastrointestinal stromal tumor: diagnosis and management. Internation Journal of Surgery Research Practice. 2019; 6: 93

[7] El-Menyar A, Mekkodathil A, Al-Thani H. Diagnosis and management of gastrointestinal stromal tumors: an up-to-date literature review. Journal of Cancer Research Therapeutics. 2017; 13(6): 889-900. 
[8] Popivanov G, Tabakov M, Mantese G, Cirocchi R, Piccinini I, D'Andrea V, et al. Surgical treatment of gastrointestinal stromal tumors of the duodenum: a literature review. Translational Gastroenterology and Hepatology, 2018; 3

[9] Flores-Funes D, Lirón-Ruiz RJ, Pérez-Guarinos CV, Martín-Lorenzo JG, Torralba-Martínez JA, Giménez-Bascuñana A, et al. Clinical and pathological features of gastrointestinal stromal tumors (GIST) in a single institution: a descriptive study and review of the literature. Cirugía Española (English Edition), 2017; 95(7): 391-396.

[10] Marcella C, Shi RH, Sarwar S. Clinical overview of GIST and its latest management by endoscopic resection in upper GI: a literature review. Gastroenterology Research and Practice. 2018; 2018: 6864256.

[11] Yang J, Yan J, Zeng M, Wan W, Liu T, Xiao JR. Bone metastases of gastrointestinal stromal tumor: a review of published literature. Cancer Management and Research. 2020; 12: 1411-1417.

[12] Rodrigues JBSR, Campanati RG, Nolasco F, Bernardes AM, Sanches SR, Savassi-Rocha PR. Pre-operative gastric gist downsizing: the importance of neoadjuvant therapy. Archive of "Arquivos Brasileiros de Cirurgia Digestiva: ABCD". 2019; 32(1): 10-12.

[13] Kale SS, Sachdev MS, Ismail MK, Davila R, Tombazzi CR. A case and literature review of complicated gastrointestinal stromal tumors. Gastroenterol Hepatology. 2008; 4(9): 650-657.

[14] Madhavan A, Phillips AW, Donohoe CL, Willows RJ, Immanuel A, Verril M, et al. Surgical management of gastric gastrointestinal stromal tumours: comparison of outcomes for local and radical resection. Gastroenterology Research and Practice. 2018; 2018.

[15] Đokić M, Novak J, Petrič M, Ranković B, Štabuc M, Trotovšek B. Case report and literature review: patient with gastroduodenal intussusception due to the gastrointestinal stromal tumor of the lesser curvature of the gastric body. BMC Surgery. 2019; 19(1): 1-6.

[16] Prablek M, Srinivasan VM, Srivatsan A, Holdener S, Oneissi M, Heck $\mathrm{KA}$, et al. Gastrointestinal stromal tumor with intracranial metastasis: case presentation and systematic review of literature. BMC Cancer. 2019; 19(1): 1-7.

[17] Shah K, Chan KKW, Ko YJ. A systematic review and network metaanalysis of post-imatinib therapy in advanced gastrointestinal stromal tumour. Current Oncology. 2017; 24(6): e531-e539.

[18] Antonioli DA. Gastrointestinal autonomic nerve tumors. Expanding the spectrum of gastrointestinal stromal tumors. Archives of Pathology and Laboratory Medicine. 1989; 113(8): 831-833.

[19] Appelman HD. Smooth muscle tumors of the gastrointestinal tract. What we know now that Stout didn't know. American Journal of Pathology. 1986; 10: 83-99.

[20] Franquemont DW. Differentiation and risk assessment of gastrointestinal stromal tumours. American Journal of Pathology. 1995;(103):41-47.

[21] Mazur MT, Clark HB. Gastric stromal tumors. Reappraisal of histogenesis. American Journal of Surgical Pathology. 1983; 7(6): 507-519.

[22] Miettinen M, Virolainen M. Gastrointestinal stromal tumors--value of CD34 antigen in their identification and separation from true leiomyomas and schwannomas. American Journal of Surgical Pathology. 1995; 19(2): 207-216.

[23] Mikhael AI, Bacchi CE, Zarbo RJ, Ma CK, Gown AM. Cd34 expression in stromal tumors of the gastrointestinal-tract. Applied Immunohistochemistry, 1994; 89-93.

[24] Corless CL, Fletcher JA, Heinrich MC. Biology of gastrointestinal stromal tumors. Journal of Clinical Oncology. 2004; 22(18): $3813-$ 3825.

[25] Subramanyam B, Pond SM, Eyles DW, Whiteford HA. Fouda Stroke Therapy Academic Industry Roundtable (STAIR): Recommendations for standards regarding preclinical neuroprotective and restorative drug development. Stroke. 1999; 30(12): 2752-2758.

[26] Heinrich MC, Corless CL, Duensing A, McGreevey L, Chen CJ, Joseph $\mathrm{N}$, et al. PDGFRA activating mutations in gastrointestinal stromal tumors. Science. 2003; 299(5607): 708-710.

[27] Agaram NP, Wong GC, Guo T, Maki RG, Singer S, DeMatteo RP, et al. Novel V600E BRAF mutations in imatinib-naive and imatinibresistant gastrointestinal stromal tumors. Genes, Chromosomes and Cancer. 2008; 47(10): 853-859.

[28] Ahmed M. Recent advances in the management of gastrointestinal stromal tumor. World Journal of Clinical Cases. 2020; 8(15): 31423155 .

[29] Beham AW, Schaefer IM, Schüler P, Cameron S, Ghadimi BM. Gastrointestinal stromal tumors. International Journal of Colorectal Disease. 2012; 27(6): 689-700.

[30] Carney JA, Sheps SG, Go VL, Gordon H. The triad of gastric leiomyosarcoma, functioning extra-adrenal paraganglioma and pulmonary chondroma. New England Journal of Medicine 1977; 296(26): 1517-1518
[31] Nishida T, Blay JY, Hirota S, Kitagawa Y, Kang YK. The standard diagnosis, treatment, and follow-up of gastrointestinal stromal tumors based on guidelines. Gastric Cancer. 2016; 19(1): 3-14.

[32] Caterino S, Lorenzon L, Petrucciani N, Iannicelli E, Pilozzi E, Romiti A, et al. Gastrointestinal stromal tumors: correlation between symptoms at presentation, tumor location and prognostic factors in 47 consecutive patients. World Journal of Surgical Oncology. 2011; 9(1): $1-10$.

[33] Lim KT. Surgical treatment of gastrointestinal stromal tumors of the stomach: current status and future perspective. Translational Gastroenterology and Hepatology. 2017; 2: 104.

[34] Gervaz P, Huber O, Morel P. Surgical management of gastrointestinal stromal tumours. British Journal of Surgery. 2009; 96(6): 567-578.

[35] Tornillo L, Terracciano LM. An update on molecular genetics of gastrointestinal stromal tumours. Journal of Clinical Pathology. 2006; 59(6): 557-563.

[36] DeMatteo RP, Lewis JJ, Leung D, Mudan SS, Woodruff JM, Brennan MF. Two hundred gastrointestinal stromal tumors: recurrence patterns and prognostic factors for survival. Annals of Surgery. 2000; 231(1): 51-58.

[37] Koo DH, Ryu MH, Kim KM, Yang HK, Sawaki A, Hirota S, et al. Asian consensus guidelines for the diagnosis and management of gastrointestinal stromal tumor. Cancer research and treatment: official journal of Korean Cancer Association, 2016; 48(4): 1155.

[38] Coe TM, Fero KE, Fanta PT, Mallory RJ, Tang CM, et al. Populationbased epidemiology and mortality of small malignant gastrointestinal stromal tumors in the USA. Journal of Gastrointestinal Surgery, 2016; 20(6): 1132-1140 